TAMKANG JOURNAL OF MATHEMATICS

Volume 33, Number 3, Autumn 2002

\title{
RAMANUJAN'S REMARKABLE SUMMATION FORMULA AS A 2-PARAMETER GENERALIZATION OF THE QUINTUPLE PRODUCT IDENTITY
}

\author{
S. BHARGAVA, CHANDRASHEKAR ADIGA AND M. S. MAHADEVA NAIKA
}

\begin{abstract}
It is well known that 'Ramanujan's remarkable summation formula' unifies and generalizes the $q$-binomial theorem and the triple product identity and has numerous applications. In this note we will demonstrate how, after a suitable transformation of the series side, it can be looked upon as a 2-parameter generalization of the quintuple product identity also.
\end{abstract}

\section{Introduction}

One of the famous identities of Ramanujan is his ${ }_{1} \boldsymbol{\Psi}_{1}$ summation: If $|\beta q|<|z|<\frac{1}{|\alpha q|}$ and $|q|<1$, then

$$
\sum_{n=-\infty}^{\infty} \frac{\left(\frac{1}{\alpha} ; q^{2}\right)_{n}(-\alpha q z)^{n}}{\left(\beta q^{2} ; q^{2}\right)_{n}}=\frac{\left(-q z ; q^{2}\right)_{\infty}\left(\frac{-q}{z} ; q^{2}\right)_{\infty}\left(q^{2} ; q^{2}\right)_{\infty}\left(\alpha \beta q^{2} ; q^{2}\right)_{\infty}}{\left(-\alpha q z ; q^{2}\right)_{\infty}\left(\frac{-\beta q}{z} ; q^{2}\right)_{\infty}\left(\alpha q^{2} ; q^{2}\right)_{\infty}\left(\beta q^{2} ; q^{2}\right)_{\infty}}
$$

where

$$
\begin{gathered}
(a)_{0}:=(a ; q)_{0}=1, \\
(a)_{\infty}:=(a ; q)_{\infty}:=\prod_{0}^{\infty}\left(1-a q^{n}\right),
\end{gathered}
$$

and

$$
(a)_{n}:=\frac{(a)_{\infty}}{\left(a q^{n}\right)_{\infty}} .
$$

This appears as Entry 17 of Chapter 16 of his Second Notebook [4, p. 196]. A number of proofs and good many applications of (1.1) have been discovered since the time of $\mathrm{G}$. H. Hardy [3, pp. 222, 223] who brought it to light. In one of his books B. C. Berndt [1, p. 32] has referred to 11 papers dedicated to proofs, 8 papers containing varied type of applications and several generalizations and multidimensional analogues of (1.1). One

Received June 7, 2001; revised August 16, 2001.

2000 AMS Subject Classification. 11S23, 33D20, 05A19.

Key words and phrases. Ramanujan's remarkable summation formula, quintuple-product identity. 
of the striking aspects of (1.1) is that it is a common generalization of the well known Jacobi's triple product identity and Euler-Cauchy $q$-binomial theorem.

The following identity, known as the quintuple product identity,

$$
(-x)_{\infty}\left(\frac{-q}{x}\right)_{\infty}(q)_{\infty}\left(x^{2} q ; q^{2}\right)_{\infty}\left(\frac{q}{x^{2}} ; q^{2}\right)_{\infty}=\sum_{-\infty}^{\infty}(-1)^{n} q^{\frac{3 n^{2}+n}{2}}\left(x^{3 n+1}+x^{-3 n}\right)
$$

for $|q|<1$, and $x \neq 0$ has been discovered many times in the past. One may see, for instance, Berndt's work [1] for an account of several proofs and applications.

The purpose of this note is to bring out another feature of the remarkable formula (1.1). Aided by Rogers - Fine identity [2, p. 15], we will infact show how (1.1), after suitably transforming its series side, can be regarded as a 2-parameter generalization of the quintuple product identity (1.2).

\section{Main Result}

Theorem 2.1. If $|q|<1$ and $|\beta q|^{1 / 2}<|x|<\frac{1}{|\alpha q|^{1 / 2}}$, then

$$
\begin{aligned}
& \frac{\left(x^{2}\right)_{\infty}\left(\frac{q}{x^{2}}\right)_{\infty}(q)_{\infty}(\alpha \beta q)_{\infty}}{\left(\alpha x^{2}\right)_{\infty}\left(\frac{\beta q}{x^{2}}\right)_{\infty}(\alpha q)_{\infty}(\beta q)_{\infty}} \\
= & \sum_{0}^{\infty} \frac{(-1)^{n}\left(\frac{\alpha}{q^{n-1}}\right)_{n}\left(\frac{x^{2}}{\beta}\right)_{n}\left(1-x^{2} q^{2 n}\right)\left(\frac{\beta}{x}\right)^{n} q^{\frac{3 n^{2}-n}{2}} x^{3 n}}{(\beta q)_{n}\left(\alpha x^{2}\right)_{n+1}} \\
& -\sum_{1}^{\infty} \frac{(-1)^{n}\left(\frac{1}{\beta}\right)_{n}\left(\frac{\alpha x^{2}}{q^{n-1}}\right)_{n-1}\left(\frac{\beta}{x}\right)^{n}\left(1-\frac{q^{2 n}}{x^{2}}\right) q^{\frac{3 n^{2}-n}{2}} x^{-3 n+2}}{(\alpha q)_{n}\left(\frac{\beta q}{x^{2}}\right)_{n}} .
\end{aligned}
$$

Proof. On replacing $q$ by $q^{1 / 2}$ and $z$ by $-x^{2} q^{-1 / 2}$ the remarkable formula (1.1) can be written as

$$
\frac{\left(x^{2}\right)_{\infty}\left(\frac{q}{x^{2}}\right)_{\infty}(q)_{\infty}(\alpha \beta q)_{\infty}}{\left(\alpha x^{2}\right)_{\infty}\left(\frac{\beta q}{x^{2}}\right)_{\infty}(\alpha q)_{\infty}(\beta q)_{\infty}}=\sum_{0}^{\infty} \frac{\left(\frac{1}{\alpha}\right)_{n}\left(\alpha x^{2}\right)^{n}}{(\beta q)_{n}}+\sum_{1}^{\infty} \frac{\left(\frac{1}{\beta}\right)_{n}\left(\frac{\beta q}{x^{2}}\right)^{n}}{(\alpha q)_{n}}
$$

Denoting the first sum on the right side of (2.2) by $F(\alpha, \beta, x)$ and applying Rogers-Fine identity [2, p. 15], namely

$$
\sum_{0}^{\infty} \frac{(a)_{n} \tau^{n}}{(b)_{n}}=\sum_{0}^{\infty} \frac{(a)_{n}\left(\frac{a \tau q}{b}\right)_{n} b^{n} \tau^{n} q^{n^{2}-n}\left(1-a \tau q^{2 n}\right)}{(b)_{n}(\tau)_{n+1}}
$$


(with $|q|<1$ and $|\tau|<1$ ) we have the transform

$$
\begin{aligned}
F(\alpha, \beta, x) & =\sum_{0}^{\infty} \frac{\left(\frac{1}{\alpha}\right)_{n}\left(\frac{x^{2}}{\beta}\right)_{n} \alpha^{n} \beta^{n} q^{n^{2}} x^{2 n}\left(1-x^{2} q^{2 n}\right)}{(\beta q)_{n}\left(\alpha x^{2}\right)_{n+1}} \\
& =\sum_{0}^{\infty} \frac{(-1)^{n}\left(\frac{\alpha}{q^{n-1}}\right)_{n}\left(\frac{x^{2}}{\beta}\right)_{n}\left(1-x^{2} q^{2 n}\right)\left(\frac{\beta}{x}\right)^{n} q^{\frac{3 n^{2}-n}{2}} x^{3 n}}{(\beta q)_{n}\left(\alpha x^{2}\right)_{n+1}} .
\end{aligned}
$$

Similarly, the second sum on the right side of (2.2) can be transformed as

$$
\begin{aligned}
& \frac{q(\beta-1)}{(1-\alpha q) x^{2}} F\left(\frac{\beta}{q}, \alpha q, \frac{q}{x}\right) \\
= & -\sum_{1}^{\infty} \frac{(-1)^{n}\left(\frac{1}{\beta}\right)_{n}\left(\frac{\alpha x^{2}}{q^{n-1}}\right)_{n-1}\left(\frac{\beta}{x}\right)^{n} q^{\frac{3 n^{2}-n}{2}} x^{-3 n+2}\left(1-\frac{q^{2 n}}{x^{2}}\right)}{(\alpha q)_{n}\left(\frac{\beta q}{x^{2}}\right)_{n}} .
\end{aligned}
$$

Using (2.4) and (2.5) in (2.2), we have (2.1).

\section{Remark.}

(i) Putting $\alpha=0$, and $\beta=x$, in (2.1), we obtain

$$
\begin{aligned}
\frac{\left(x^{2}\right)_{\infty}\left(\frac{q}{x^{2}}\right)_{\infty}(q)_{\infty}}{\left(\frac{q}{x}\right)_{\infty}(x q)_{\infty}} & =\sum_{-\infty}^{\infty}(-1)^{n}\left(1+x q^{n}\right) q^{\frac{3 n^{2}-n}{2}} x^{3 n} \\
& =\sum_{-\infty}^{\infty}(-1)^{n} q^{\frac{3 n^{2}+n}{2}}\left(x^{3 n+1}+x^{3 n} q^{-n}\right),
\end{aligned}
$$

which is same as the quintuple product identity (1.2).

(ii) The identity (2.1) contains other elegant special cases also namely the case $\beta=x$ or $\alpha=0$.

\section{Acknowledgement}

The authors are grateful to Prof. H. M. Srivastava for his suggestions which considerably improved the quality of this note.

\section{References}

[1] B. C. Berndt, Ramanujan's Notebooks, Part III, Springer-Varlag, New York, 1991. 
[2] N. J. Fine, Basic Hypergeometric Series and Applications, American Mathematical Society, Providence, 1988.

[3] G. H. Hardy, Ramanujan, 3rd ed, Chelsea, New York, 1978.

[4] S. Ramanujan, Notebooks (Volume 2), Tata Institute of Fundamental Research, Bombay, 1957.

Department of Studies in Mathematics, University of Mysore, Manasagangothri, Mysore-570 006, India. 